\title{
¿Puede construirse "actitud cooperativa" en los participantes de un sistema educativo con un enfoque de competencias?
}

\author{
Ventura Alfonso Alas ${ }^{1}$ \\ Herman Van de Velde ${ }^{2}$
}

\section{RESUMEN}

Cuando las personas participamos en procesos de formación llevamos cierta actitud o prácticas individualistas que han sido fomentadas por el sistema educativo y el tipo de sociedad en el que vivimos. Esta actitud se refleja no solamente en nuestros procesos formativos personales sino también en las dinámicas, enfoques y formas de relacionamiento social que establecemos, tanto en el ámbito familiar, laboral o comunitario. La sociedad, hoy en día, con todo su sistema comercial, nos ha empujado a competir entre nosotras/os, justificándose en la consecución de metas cada vez más elevadas. Este modelo también está presente en los contextos educativos: se fomenta la competitividad tanto por parte de las familias, que esperan el éxito escolar, como por parte de la propia escuela o comunidad donde se trabaja la mayor parte del tiempo de forma individual. Este artículo somete a una revisión crítica el sistema de educación salvadoreño con enfoque de competencias, pero a la vez se propone que los procesos de aprendizaje trasciendan las paredes de la escuela y se establezcan relaciones horizontales entre los participantes, mediante la práctica cotidiana de la "cooperación genuina", dicho paradigma se convierta en un estilo de vida.

Palabras clave: Sistema educativo, Competencias, Cooperación genuina.

Recibido: 10 de diciembre de 2014

Aceptado: 10 de marzo de 2015

1 Licenciado en Ciencias de la Educación y profesor de Ciencias Sociales con estudiantes de Tercer Ciclo de Educación Básica en el Centro Escolar San Antonio Los Ranchos. Representante de ABACOenRed en El Salvador. Correo Electrónico: venturalas@yahoo.com

2 Doctor en Ciencias Pedagógicas, docente UNAN-Managua /FAREM-Estelí y coordinador de ABACOenRed (www. abacoenred.com), un referente nuestroamericano como propuesta pedagógico-metodológica en construcción. Correo Electrónico: herman@abacoenred.com 


\title{
Can "cooperative attitude" be built in participants of an education system with a focus on competencies?
}

\begin{abstract}
When people participate in training processes they carry certain attitudes or individualistic practices that have been fostered by the educational system and the type of society in which we live. This attitude is reflected not only in our personal training processes but also in dynamics, approaches and forms of social relationships we establish, in the family, community or labor. Society today, with all its trading system, has pushed us to compete among us, justified in the pursuit of ever higher goals. This model is also present in educational contexts: competitiveness is encouraged both by families who expect school success and by the school itself or community where time working is the most spent individually. This article subjects to critical review the Salvadoran education system with a focus on competencies, but also proposes that the learning processes transcend the school walls and that horizontal relationships between participants are established through the daily practice of " genuine cooperation ", whose paradigm becomes a lifestyle.
\end{abstract}

Keywords: Educational Systems, Competencies, Genuine Cooperation. 


\section{INTRODUCCIÓN}

Enrepetidasocasiones, elsistemaeducativosalvadoreño ha experimentado cambios de tipo estructural y a veces de forma, todos esos cambios han desaparecido tal cual como fueron propuestos e impuestos por los gobiernos de turno, el último enfoque ha sido el de una educación basada en competencias; un enfoque que al ser revisado con ojo clínico resulta ser muy dañino para la sociedad. La degradación del medio ambiente, es una muestra de ello, el alto grado de deshumanización por la práctica de antivalores, la falta de conciencia social por los problemas de las personas.

Este artículo busca hacer un paralelo entre la educación basada en competencias y los procesos de aprendizaje basados en actitudes cooperativas, cooperación que se basa en 5 pilares fundamentales: escuchar, interpretar, contextualizar, compromiso de cooperar y tener un posicionamiento claro de las implicaciones pedagógicas y políticas de la educación; estos 5 pilares deben vertebrarse a través de una confianza mutua entre los participantes y la capacidad de crítica y autocrítica constructiva.

Finalmente se exponen las razones que nos deben llevar a construir una actitud cooperativa, así como los elementos que implica, para llegar a establecer algunas conclusiones sobre lo que ha versado este artículo.

\section{MATERIALES Y MÉTODOS}

Investigación bibliográfica

\section{RESULTADOS Y DISCUSIÓN:}

En más de una oportunidad se ha escuchado a padresmadres de familia diciendo a sus hijos/as: "vamos a ver quién termina primero de comer, quien lo consiga ganará un premio". En los deportes, aunque sea a nivel comunitario, en el campo futbolero del barrio, se escuchan expresiones de ganar y perder, el empate no gusta a ninguno de los dos equipos, ganar representa un peldaño psicológico superior al ganador y un peldaño inferior al perdedor. En las escuelas se premian las "buenas calificaciones" y se castigan las "malas", se jerarquizan a los estudiantes etiquetándolos en primer lugar, segundo lugar, tercer lugar...

En el ámbito económico, las empresas compiten entre sí para posicionar sus marcas, para vender más sus bienes y servicios, no importa si en ese proceso hacen desaparecer otras iniciativas económicas que también tengan el derecho de existir y operar. Internamente, las instituciones empresariales impulsan procesos en el cual hacen competir a sus trabajadores para ganar un incentivo económico, para mejorar las relaciones con el jefe/a u obtener un puesto de trabajo determinado.

En diferentes contextos, la competencia parece haberse incorporado como estilo de vida en muchas sociedades del mundo. El éxito justifica la competencia. Vivimos en una etapa de la historia donde la aritmética básica es ganar-perder, no hay espacios donde haya equidad e igualdad, donde la relación sea ganar-ganar. Aún sabiendo que quien gana, lo hace aprovechando el fracaso de los demás, un fracaso que en muchas ocasiones es auspiciado por sus contrincantes más cercanos; así surgen las rivalidades, los adversarios...

En la competitividad hay rivalidad por ser el mejor, envidia de los triunfos ajenos, individualismo al querer todo para uno y nada para los demás, una alta dosis de egocentrismo para hacer girar todas las posibilidades de triunfos sobre uno mismo; desánimos al sentirse inferior ya que la competencia conlleva a derrotas, ofensas hacia los demás como estrategia de dejarlos a medio camino... ¿Soñamos con PerSociales ${ }^{1}$ con un

1 Enfoque 'PerSocial' referido a nuestro contexto personal, familiar, social y comunitario. Se trata de un enfoque donde dejamos de separar lo personal y lo social, considerando que se trata de una UNIDAD (integrada) inseparable. Se basa en la filosofía de UBUNTU: 'Soy porque Somos'. En ABACOenRed se ha ampliado esta expresión para fortalecer el sentir de 'proceso' y dice: "Soy siendo porque Somos siendo." (Herman en el Glosario del curso 'Formulación de micro-proyectos sociales) 
paradigma de vida competitivo? ¿Es este es el estilo de sociedad al que aspiramos?

Las diferentes expresiones competitivas en la sociedad, en los diversos espacios de convivencia, responden a un orden económico y social internacionalmente establecido e impuesto. Las libertades económicas exigen competitividad para su buen funcionamiento, la globalización de las grandes economías mundiales abogan por competir. En esta misma dirección, muchos gobiernos han llevado el enfoque competitivo empresarial hasta el seno del funcionamiento estatal, a tal grado que han llegado a incorporar enfoques de educación basados en competencias. El Salvador, que será el contexto geográfico donde se centrará este análisis, es uno de los países con este enfoque. ¿Podemos esperar comportamientos distintos en la población cuando el sistema educativo promueve la competencia? Si se impulsa un currículo que prepara para la vida, significa entonces que estamos preparando para la competencia. En el Ministerio de Educación como ente rector y regulador, descansa la formación del futuro generacional del país.

Retomando el papel de la educación, Humberto Vandenbulcke $^{2}$ (2013) reflexiona en el foro de LinkedIn ${ }^{3}$ sobre métodos y técnicas de aprendizaje cooperativo en ambientes virtuales, plantea que no es que se dude del rol de la educación en procesos de cambio, sino que considera muy importante "tener claro el modelo de sociedad al que aspiramos, que soñamos, que deseamos y que pretendemos ayudar a construir, entre otras mediante un modelo de educación". Según él, si aspiramos a una sociedad más solidaria, igualitaria, ética e incluyente nuestro modo de educar debe distanciarse radicalmente del paradigma bancario, el modelo de la imposición, de la importación de

2 Profesional de origen Belga, radicado actualmente en República Dominicana quien es consultor Community Radio communication. Ha sido secretario ejecutivo de UDECA (1996-2002); director nacional de Fe y Alegría para República Dominicana (2008-2009) y actualmente es coordinador del proyecto ALER2020.

3 Red Social orientada a establecer relaciones profesionales e intercambio de ideas sobre temas diversos verdades universales. Nuestro modelo educativo no será la pedagogía de la respuesta (impuesta) sino de la pedagogía de la pregunta, que es capaz de sacar todo el potencial de cada persona, en su capacidad de entender y profundizar su realidad y contexto y también en su capacidad de generar, junto con otros/as, repuestas originales para su vida y de su comunidad.

Los titulares del MINED ${ }^{4}$ defienden el argumento de la necesidad de entrar en una dinámica de enfocar el sistema educativo a las competencias, defendiendo la tesis que "Con el auge de un mundo globalizado, el acceso veloz de la información y la tecnología; el aprendizaje por competencias adquiere relevancia mundial". (MINED 2008, p 7) Currículo al servicio del aprendizaje.

Las corporaciones empresariales exigen que el sistema educativo responda a sus demandas, hay una lógica entonces, el orden económico determina el modelo educativo que debemos adoptar; aunque debería ser lo contrario, que el modelo educativo definiera el modelo económico a desarrollar. Parece que hay una preocupación por parte del gobierno por capacitar mano de obra: operadores de maquinaria industrial, el dominio de un idioma determinado, con énfasis en el inglés, la habilidad para manejar un ordenador.

De acuerdo al Currículo al servicio del aprendizaje (2008, p 10 y 11) documento que guía, orienta y regula el sistema educativo en El Salvador ratifica esta idea expresando literalmente entre otras, las siguientes ventajas de trabajar por competencias.

1. "Estimula la actualización contínua de los programas educativos, para poder responder a las necesidades reales de los educandos en la sociedad globalizada y del avance de la ciencia y la tecnología.

2. Se adapta a la necesidad de compartir esquemas diversos de formación presentes en la sociedad internacional.

3. Se vincula la educación con las demandas requeridas en el mundo laboral..."

4 Ministerio de Educación de El Salvador. 
La balanza está inclinada en la preparación de obreros/as y no en la formación de PerSociales, hay una preocupación por la globalización, por las comunicaciones, por el uso de la tecnología.

La filosofía del currículo nacional estriba en que "Ser competente es ser capaz de resolver con éxito una tarea en situaciones concretas". (MINED 2008, p 7) Curriculo al servicio del aprendizaje. ¿Y quien no consiga resolver esta tarea con éxito, será incompetente, inepto, inútil, incapaz? ¿A quiénes de los lectores/ as de este artículo nos gustaría que nos adjudiquen directa o indirectamente adjetivos calificativos de no competente? En torno a este escenario es que recobra relevancia este análisis, debido a que una educación basada en actitudes cooperativas, la prioridad es trabajar todos/as por un objetivo común y llegar juntos/as a la meta previamente establecida. Se trata de construir colectivamente oportunidades de aprendizaje, las cuales exigen una responsabilidad colectiva y una responsabilidad individual, es decir; responsabilidad compartida.

También se considera que ha habido un salto ilícito del enfoque competitivo, al traspolarlo del ámbito comercial al ámbito social-educativo. La actividad humana y específicamente los procesos de aprendizaje no deben ser contaminados con enfoques de depredación, ya que la esencia de su actividad es la formación de PerSociales que sepamos relacionarnos bien unos con otros, que tengamos buena relación con el medio ambiente, que seamos solidarios/as ante situaciones específicas y generales de la sociedad. No se está tratando de justificar la competencia en ninguna de las actividades humanas, ya que en todas sus expresiones tiene los mismos efectos: superioridadmarginación, ganar-perder, existir-desaparecer...

¿Por qué no cooperar genuinamente entonces? La cooperación no es algo nuevo, desde los pueblos prehispánicos en América, esta práctica era cotidiana, un estilo de vida que incorporaba no solamente lo social, sino la relación con el medio ambiente.
Al estudiar algunas culturas de los pueblos originarios africanos o latinoamericanos es notable encontrarnos con frases centradas en la unidad y la cooperación como opción de comunidad. En la cultura africana así como en la cultura pre-colombina latinoamericana se rescatan frases que expresan una declaración humana y esencial: la necesidad de la cooperación.

Retomando a Bautista, J. (s.f.) $)^{5}$ el saludo maya "IN LAK' ECH", que significa "yo soy otro tú", todos somos uno mismo, y se contesta: "HALA KEN": "tú eres otro yo" invita a reconocer al otro(a) y comprender que nos complementamos en la temporalidad de nuestras circunstancias, siendo una realidad fundamental e ineludible. La expresión aquí recoge la conciencia de ser iguales y que integramos un único organismo: "los otros son parte mía y yo soy parte de ellos".

Para los Mayas, el reino mineral, vegetal, animal y toda la materia del universo, "desde un átomo hasta una galaxia, son seres vivos con conciencia evolutiva". Al vivir en unidad, si eres feliz, yo soy feliz y viceversa. Porque "yo soy tú" y "tú eres yo" y cualquier cosa que me haces a mí, te la haces a ti. La cosmovisión expresada en el lenguaje, en tradiciones y en el saludo reconoce al otro como nuestro yo, desprendiendo solidaridad, respeto, unión y armonía.

Con el pasar de la historia, hemos sido testigos/as de muchas ocasiones en que solo ha sido posible superar adversidades gracias a la solidaridad y cooperación entre las personas, entre las comunidades, entre los pueblos, entre los países: guerras, terremotos, inundaciones, crisis económicas... Si en esos momentos se respondía con egoísmo y competencia, seguramente no se habría podido superar tales crisis.

Por eso, existe una necesidad latente e impostergable de reinventar un nuevo paradigma educativo que privilegie las relaciones de cooperación genuina y no la competencia entre los participantes, pero esta

5 Reflexión encontrada en http://www.taringa.net/posts/ solidaridad/15309332/Yo-soy-otro-tu---Tu-eres-otro-yo.html 
cooperación debe trascender además a las diferentes instituciones del estado y privadas con una apuesta clara y decidida al tema educativo.

Luz Mariela Avruj ${ }^{6}$ (2013) en un foro abierto en LinkedIn sobre el tema cooperar o colaborar (febrero - mayo 2013), retoma a Mario Rovere (argentino, especialista en redes) y dice que:

Cooperar es compartir actividades o recursos lo cual requiere y genera solidaridad compartiendo un objetivo común. Tener un objetivo común conlleva, además, compartir actividades o recursos para tal fin. De acuerdo a lo dicho por Avruj, la cooperación genera solidaridad y se trabaja por un objetivo común construido colectivamente, al menos en la forma que se describe para la física cuando dice que dos cuerpos son solidarios cuando nada que le ocurra a uno le es indiferente al otro.

Herman Van de Velde (2013), quien participó en este mismo foro virtual en LinkedIn, nos aporta su definición sobre cooperar:

Cooperar, implica un trabajo conjunto en el cual se comparten objetivos. Se trata de dos instancias (personales o comunitarias) que tienen sus propios objetivos y estrategias, y que encuentran un ámbito en el cual se necesitan mutuamente (interdependencia positiva) y deciden cooperar, es decir trabajar juntas/os; cada quien con su objetivo, desde sus propios intereses y en lo particular (el ámbito de la cooperación) también compartiendo un interés, un objetivo muy particular, además formulado en conjunto.

Los hermanos Johnson (1999:4) dicen que cooperar significa "trabajar juntos para lograr objetivos compartidos". Según ellos, en las actividades cooperativas, las/os integrantes de un equipo buscan productos que resulten beneficiosos para sí mismas/ os y, al mismo tiempo, para todas/os las/os otras/os integrantes del grupo.

6 Profesional argentina, facilitadora de procesos educativos.
En su artículo "Cooperar - ¡un acto esencialmente educativo!" Van de Velde (2012:3) rescata el pensar de Botello $(2004,1)$ cuando considera el concepto de cooperación como manifestación actitudinal de solidaridad, hacia la solución de necesidades o problemas comunes. Esta solidaridad, como base de la cooperación, tiene que tener una relación de horizontalidad de ambas vías lo que implica un profundo respeto mutuo.

Podemos visualizar con claridad que todos las definiciones en torno a la cooperación tienen elementos en común muy marcados: solución de necesidades comunes, establecimiento de objetivos y metas de forma colectiva, trabajar juntos/as por lograr objetivos compartidos, expresión de solidaridad. De acuerdo a los aportes, la cooperación no se reduce a un contexto determinado o a un escenario concreto; ésta va más allá de un período lectivo, de la ejecución de un proyecto, de la resolución de un conflicto; aunque sean estos los espacios donde se genera la necesidad de cooperar y donde se potencia, pero la cooperación debe traducirse en un aprendizaje, en una actitud, en un estilo de vida, en un modo de operar, en un paradigma institucional, social-comunitario.

Siguiendo a Van de Velde, la cooperación, sea como estrategia educativa, como expresión de economía solidaria o en el contexto de la cooperación (local, nacional, internacional) debe considerar los siguientes aspectos, válidos en cada contexto particular:

- El ejercicio consciente y permanente de solidaridad (responsabilidad y equidad).

- El ejercicio del diálogo sincero, entre pares, entre 'iguales' sin pre-condicionamientos y sin hipocresía.

- La identificación con un interés general, la que permite la integración social, la integración entre las partes involucradas, entre las y los actoras/es relacionadas/os en función de un proyecto políticoideológico (participación ciudadana y compromiso comunitario). 
- El respeto profundo por el aporte de cada uno de los actores involucrados. Que como también plantea Erich Fromm es tener "la capacidad de ver a una persona tal cual es... preocuparse porque la otra persona crezca y se desarrolle tal como es..."

- Identificación de una coincidencia en intereses, sobre la base de valores éticos.

- La consideración de la diversidad como una oportunidad para el aprendizaje y el desarrollo, resultando en un ejercicio activo y constructivo de interculturalidad.

- El sentimiento de necesidad de aprendizaje y desarrollo desde cada uno de los actores involucrados, sin excepción (apoyo mutuo).

Después de revisar varias concepciones sobre la cooperación, así como alguna caracterización general; se visualiza claramente que tienen puntos en común entre los diferentes autores: el establecimiento colectivo de objetivos es uno de ellos, así como trabajar juntos/ as por ese mismo objetivo previamente acordado.

A partir de lo reflexionado anteriormente, y para efectos de este artículo se decide utilizar el término cooperar y cooperación vinculado al aprendizaje, con la siguiente acepción:

La cooperación, es un proceso de construcción colectiva que busca potenciar las habilidades socioafectivas de quienes participan en un grupo y del grupo en sí mismo. Cooperar implica poder y responsabilidad compartida e interdependencia positiva.

Cooperar invita a un involucramiento emocional $y$ afectivo, en una relación horizontalidad, respeto, apoyo mutuo y dialogicidad.

La cooperación es también una actitud o forma de vida.

Y para reforzar el concepto de cooperación que se propone antes, se va a utilizar el término “Cooperación
Genuina" como un acto educativo y una acción político-pedagógica-metodológica, tal y como lo fundamenta ABACOenRed ${ }^{7}$. En este acto educativo se perfilan cinco pilares fundamentales desde su interrelación e integración creativa:

1. El arte de escuchar (solidaridad, diversidad, respeto, interculturalidad, diálogo, coincidencia,...) (apertura). No hay posibilidad de cooperar genuinamente ente diferentes instancias, ya sean personales o institucionales si no hay capacidad de escucharse; escucharse unos a otros/as, pero también escucharse uno mismo/a. Se debe dar la oportunidad de conocerse uno a otro en su contexto personal y social, con un enfoque PerSocial. Esta es la puerta de entrada para el segundo pilar.

2. La habilidad de interpretar (contextualizar, respeto a la diversidad particular según el contexto, el aporte desde abajo,...) (lectura). Interpretar el contexto en el que estamos, el punto de partida para esta cooperación genuina, el contexto de las diferentes instancias implicadas directa e indirectamente, incluyendo el contexto propio, a nivel interno y externo. Este pilar busca contextualizar, de modo que se evite la imposición.

3. La voluntad de compartir (solidaridad, respeto, diálogo, integración, interculturalidad, necesidad de aprendizaje y desarrollo, actitud de servicio,...) (ternura). Si una de las partes no tiene voluntad de compartir, la cooperación genuina no se puede desarrollar. El hermetismo, los recelos... no contribuyen para este proceso abierto y transparente.

4. La decisión de compromiso (solidaridad, integración, interculturalidad, (co-responsabilidad, actitud de servicio,...) (postura). Es un compromiso serio conmigo mismo/a, con los demás, con la comunidad. Es una decisión política.

5. La visión de integración (coincidencia en intereses, solidaridad, responsabilidad,...) (contextura). El punto de partida es que "yo solo/a no puedo", necesitamos integrarnos para poder trabajar y

7 Van de Velde (2013). RETO para la Educación en Nicaragua 2013: construir calidad educativa, basada en cooperación genuina. 66 
avanzar. De hecho, la cooperación genuina nunca será un acto solitario, es un acto colectivo, una inter-acción entre personas.

Estos 5 pilares son fundamentales porque sustentan la esencia de la cooperación genuina, pero además, deben necesariamente entrelazarse a través de dos ambientesdimensiones:

a. CONFIANZA, en uno mismo/a y en los demás.

b. CAPACIDAD DE (AUTO-) CRÍTICA CONSTRUCTIVA, tener consciencia que todos/as cometemos errores, pero cometer un error no es lo más grave, sino no reconocerlo y no superarlo.

En repetidas ocasiones he escuchado a colegas docentes quejarse del sistema educativo, del orden económico establecido, de los grandes medios de comunicación masiva, de los bajos salarios magisteriales, de la desintegración familiar, de sus compañeros/as mismos/ as que no prepararon bien a los estudiantes en grados anteriores... Traigo a cuenta estos elementos, porque la actitud implica al menos 15 dimensiones, las cuales incluyen algunas de las mencionadas anteriormente.

¿quién o quiénes son los responsables de construir una actitud cooperativa en el seno de los procesos de aprendizaje? Curiosamente cuando hay fracaso escolar, que con frecuencia se identifica el aprender con 'conocer'. Se homologa aprendizaje con conocimiento, limitándolo a una cantidad de contenidos almacenados. Se compara al ser humano con una computadora donde caben miles de datos que podemos reproducir con un solo clic. Allí ya estará la supuesta prueba del haber aprendido, como que también la computadora aprende.

En estos casos, inicia una ruleta rusa a buscar responsables: los docentes se quejan de la poca o nula presencia de los padres-madres de familia en el proceso de aprendizaje de sus hijos/as, así como de los pocos hábitos de estudio y desinterés por parte de los estudiantes; éstos a la vez reclaman por los pocos espacios que tienen para estudiar y de clases no entendibles, de poco dinamismo; los padres-madres de familia demandan profesores/as mejor formados/as y alegan que las leyes no les permite presionar a sus hijos/as para educarlos mejor. Así los alegatos de cada uno de los miembros de la comunidad educativa, uno responsabiliza al otro. A nivel institucional, el docente de un grado le tira la pelota al colega que atendió a los estudiantes en el grado anterior y éste al otro... así sucesivamente. La capacidad de crítica y autocrítica es indispensable en este ir y venir del proceso educativo; se trata de asumir una responsabilidad compartida.

Se debe cambiar el ritmo vertiginoso del hacer de "hoy y mañana, y mañana por la mañana", esta rutina no permite detenerse un rato a pensar, aunque se afirma que lo que se hace es "diferente", al cabo del tiempo se sigue haciendo lo que se sabe hacer y lo "diferente", no deja de ser otra cosa que una moda en el discurso, una palabra que trata de ocultar la repetición de lo mismo: otro empaque para el mismo producto, otros odres para el mismo vino.

La Real Academia Español (RAE) ${ }^{8}$ De acuerdo a la RAE, uno de las 3 definiciones sobre la actitud es el estado del ánimo que se expresa de una cierta manera (como una actitud conciliadora).

La actitud también ha sido definida como un estado de la disposición nerviosa y mental, que se organiza a partir de las vivencias y que orienta o dirige la respuesta de un sujeto ante determinados acontecimientos.

Según Luis Recalde, la actitud, ha sido una de las categorías más estudiadas desde la psicología social y retomando a Secor y Backman (1964) la define como "cierta regularidad en los sentimientos, pensamientos y predisposiciones del individuo para actuar en relación con algún aspecto de su ambiente".

Revisando más literatura sobre el tema de la actitud, nos encontramos con el referente metodológico de

8 Definición de actitud - Qué es, Significado y Concepto en http://definicion.de/actitud/\#ixzz2bfrr3kjU 
ABACOenRed (PIAF-P-COA_acem_c ${ }^{9}$ ) que define la actitud no solamente como una expresión externa, a nivel corpóreo, sino lo que cada persona es: el SER en sí mismo con toda su complejidad material e inmaterial e identidad PerSocial.

ABACOenRed define el aprender como cambio de actitud, ampliando sus componentes a 15 ó más, ya que defiende que "en nuestros aprendizajes no sólo se involucran nuestros pensamientos, o sentimientos, o en la evidencia del cambio de conducta, sino que también influye nuestro estado de ánimo (lo bioenergético), nuestros paradigmas éticos, el disfrute (lo estético), el arte, lo político..." (Van de Velde, 2013:5).

Nocabedudasobrelaimportanciaquetienenlas actitudes en todo proceso de aprendizaje, independientemente de su contexto y de sus particularidades. Van de Velde (2013:8) en su artículo "Cooperación genuina: un sueño a concretar que implica educación alternativa" dice que este cambio de actitud se expresa, al menos en tres sentidos:

1. Las actitudes forman parte del CONTENIDO de todo proceso de aprendizaje.

2. Las actitudes juegan un papel determinante, como INSUMO, en el mismo desarrollo del proceso de aprendizaje.

3. El cambio de las actitudes son el reflejo de los RESULTADOS de un proceso de aprendizaje.

\section{CONCLUSIONES Y RECOMENDACIONES}

Existe una necesidad latente en sectores amplios de la sociedad por desarrollar procesos de educación alternativos, en donde el objetivo principal no sea formar académicamente a los estudiantes y prepararlos para el mundo del trabajo; tampoco impregnar de miedo recorriendo el sistema educativo para hacer personas "obedientes"; sino apostar por un paradigma que prepare PerSociales independientes y creativos.

Según, Cristóbal Suárez: "la escuela no requiere inventar la cooperación, lo que necesita es asumirla - e implicarla en los modelos pedagógicos- como condición social de aprendizaje" (2008: 15). Para este autor debemos ser conscientes que la cooperación, como estrategia de desarrollo humano, no requiere mucha presentación, es tan antigua como la humanidad que la ha validado en innumerables ocasiones para subsistir, progresar y sobre todo, configurar lo propiamente humano con otros, la cultura. Se trata más bien entonces de operativizarla. Hacer caminar este proceso implica necesaria un cambio de actitud, una actitud cooperativa.

Construir actitud cooperativa implica responsabilidad compartida de toda la comunidad educativa, no resulta de la suerte o por el accionar de uno de sus miembros; aunque hay que reconocer que el facilitador/a está llamado a iniciar más temprano que tarde este proceso. Debe necesariamente generarse oportunidades de aprendizajes colectivas, aunque su aprovechamiento sea de carácter individual. En este proceso colegiado, las relaciones deben ser horizontales, un poder compartido, así como dar y recibir simultáneamente; la aritmética debe ser ganar-ganar.

9 Paradigma Integrador del Aprendizaje y su Facilitación - y su concresión metodológica el P_COA_acem_c, que significa "Procesos de Construcción Colectiva de Oportunidades de Aprendizajes, basados en una actitud emprendedora de calidad cooperativa creciente. Según lo dicho en el documento "cooperación genuina: un sueño a concretar que implica educación alternativa" de Herman Van de Velde, 2013, a partir de este nombre P_COA_acem_c se plantea "la necesidad de concebir el proceso educativo como un proceso de construcción colectiva de oportunidades de aprendizajes. En este proceso se refleja, se expresa la esencia de la interrelación e interacción social en un contexto histórico-sociocultural que sustenta el desarrollo humano personal-comunitario". Al hablar de un 'referente metodológico' se refiere a un conjunto organizado de pautas metodológicas sustentadas en principios pedagógicos y didácticos que responden a un 'paradigma integrador del aprendizaje y su facilitación' (PIAF). 


\section{BIBLIOGRAFÍA}

http://definicion.de/actitud/\#ixzz2bfrr3kjU revisado por última vez el 06 de septiembre de 2013.

http://www.antonimos.net/competente

http://www.innovacioncontinua.com/aprendizaje colaborativo.html revisada por última vez el 06 de septiembre de 2013.

http://www.taringa.net/posts/solidaridad/15309332/
Yo-soy-otro-tu---Tu-eres-otro-yo.html revisado por última vez el 05 de septiembre de 2013.

Van de Velde (2013). RETO para la Educación en Nicaragua 2013: construir calidad educativa, basada en cooperación genuina. Estelí: ABACOenRed.

Van de Velde, H. (2010). Cooperar - iun acto esencialmente educativo! Estelí: ABACOenRed. 